\title{
A Comparison of the Flipped Classroom Model for Medical Student Education in Ophthalmology before and during the COVID-19 Pandemic
}

\author{
Kelly H. Yom, BA ${ }^{1} \quad$ Ryan J. Diel, MD ${ }^{1} \quad$ Pavlina S. Kemp, MD ${ }^{1}$ \\ ${ }^{1}$ Department of Ophthalmology and Visual Sciences, University of \\ lowa Hospitals \& Clinics, lowa City, lowa \\ J Acad Ophthalmol 2021;13:e228-e233. \\ Address for correspondence Pavlina S. Kemp, MD, Department of \\ Ophthalmology and Visual Sciences, University of lowa Hospitals \& \\ Clinics, 200 Hawkins Drive, lowa City, IA 52242 \\ (e-mail: pavlina-kemp@uiowa.edu).
}

\begin{abstract}
Keywords

- flipped classroom

- ophthalmology

- medical student education

- COVID-19

- pandemic

- virtual learning

- online learning
\end{abstract}

Background The coronavirus disease 2019 (COVID-19) pandemic has necessitated a shift of medical education to online learning in lieu of traditional clinic-based rotations. The flipped classroom model, which has been shown to be an effective approach to the medical student ophthalmology curriculum, shows promise as a method of learning that can be shared virtually. As such, the aim of this study was to investigate the effectiveness of a flipped classroom approach to the primary care-focused ophthalmology clerkship delivered entirely online in the face of the COVID-19 pandemic.

Methods Medical students who completed the 2-week introductory clinical ophthalmology clerkship from July 2019 to July 2020 were included in this study. The curriculum centers on a flipped classroom model and was delivered in person prior to March 2020, after which it transitioned to an online format for 5 months. A survey was administered to students completing this rotation, which utilized 6-point Likert scales to assess students' interest in the field of ophthalmology, perceptions of the flipped classroom curriculum, and confidence in evaluating ophthalmic complaints. The cohort that completed in-person learning was then compared with the cohort that completed online learning.

Results A total of 112 responding students were included in our analysis, with 68/82 students from the in-person cohort and 44/66 students in the online cohort responding to the survey. Students who participated in the online virtual clerkship were equally likely to feel comfortable evaluating eye complaints than those who participated in the in-person clerkship, and more likely to report that the course enhanced their interest in ophthalmology. However, online students were less likely to feel comfortable using the direct ophthalmoscope compared with those in the in-person cohort.

Conclusion Regardless of its mode of delivery, the flipped classroom model is able to impart confidence in evaluating ophthalmic complaints and interest in the field of ophthalmology to medical students. While online learning can never serve as a full received

September 24, 2020 accepted after revision February 3, 2021
DOI https://doi.org/ $10.1055 / \mathrm{s}-0041-1740397$ ISSN 2475-4757.

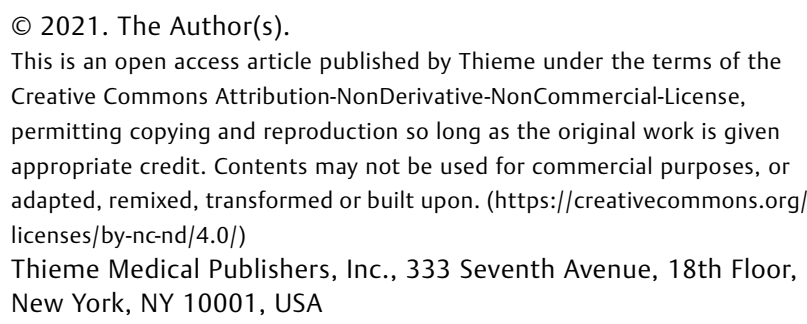
appropriate credit. Contents may not be used for commercial purposes, or adapted, remixed, transformed or built upon. (https://creativecommons.org/ licenses/by-nc-nd/4.0/) Thieme Medical Publishers, Inc., 333 Seventh Avenue, 18th Floor, New York, NY 10001, USA 
replacement for learning physical examination skills and other important clinical experiences, there are aspects of the virtual flipped classroom that do not compromise student's learning and engagement and may be implemented into curricula even after clinical activities are allowed to resume.

With the enactment of social distancing mandates and the need to preserve resources such as personal protective equipment, the coronavirus disease 2019 (COVID-19) pandemic has significantly disrupted medical education, especially for students in the clinical phase of their training. In the face of this pandemic, the Association of American Medical Colleges issued an official recommendation in March 2020 for medical schools to temporarily suspend medical students' participation in any activities that involve direct patient contact. ${ }^{1}$ As a result, many academic activities have transitioned to online formats instead of traditional clinical rotations in an effort to minimize in-person contact, slow the spread of disease, and preserve personal protective equipment. This includes rotations in ophthalmology, further decreasing the already limited clinical exposure that medical students have in this field.

Despite the challenges that online learning brings, this pandemic also allows for the opportunity to trial novel and innovative models for education, as well as the evaluation of existing models in new contexts. In fact, COVID-19 may serve as the catalyst for revolutionizing aspects of medical education that have remained stagnant for decades. ${ }^{2}$ As more educational content is converted to online formats, documenting and investigating the student's response to online learning are important to understanding how educators can incorporate the elements that are effective and improve those that are not.

One area of promise in online learning is the flipped classroom, in which learners engage with curricular material prior to class, allowing class time to be used for group discussion and problem-based application of the acquired knowledge. This is in contrast to the traditional classroom, which relies on class time to deliver lectures. The flipped classroom has already been shown in the literature to be effective for medical students both in general and within ophthalmology education, as it promotes active learning and allows students to consume lecture content at an individualized pace. ${ }^{3-6}$ Previous studies also confirm that the flipped classroom model is generally preferred by learners and educators alike over traditional models. ${ }^{6-8}$ The flipped classroom lends itself elegantly to the online format, as the prerecorded videos can be watched remotely at the student's own pace, and discussions can be held via videoconferencing without sacrificing the ability to engage in case-based education.

Previous work by our team demonstrated the successful implementation of a flipped classroom model for ophthalmology at the University of Iowa Carver College of Medicine, with increased student's satisfaction across multiple aspects of the learning experience and comparable examination performance compared with the prior lecture-based modality. ${ }^{9}$ However, it remained to be answered what aspects of this flipped classroom would transfer well to a purely virtual model as necessitated by the COVID-19 pandemic. As such, we sought to quantify the effect that a purely online flipped classroom ophthalmology curriculum would have on student learning, confidence with primary care level ophthalmology skills, and interest in the field.

\section{Methods}

\section{Curriculum Structure}

The introductory clinical ophthalmology clerkship at the University of Iowa is a 2-week long selective rotation available to medical students who have completed their third semester of medical school. The curriculum was transitioned, in 2017, to a chief-complaint-based flipped classroom model with a primary care focus. At the beginning of the clerkship, students are given access to a series of 10 prerecorded online video lectures which have been prepared by the clerkship director of medical student education, as well as the corresponding lecture slide sets and the American Academy of Ophthalmology (AAO)'s Basic Ophthalmology textbook. ${ }^{10}$ Each lecture is assigned to a specific day of the rotation, and students are expected to watch the lecture, view the slide set, or read the textbook chapter corresponding to that day's topic before participating in the group learning session. This group session consists of case-based discussion of topics from the AAO textbook, led by faculty, fellows, or residents. All content, including the videos, casebased discussions, and virtual curriculum used can be found on EyeRounds.org at: https://eyerounds.org/article/onlineophthalmology-curriculum/index.htm.

Prior to the COVID-19 pandemic, students met each morning for their in-person discussion session, and then generally spent the rest of the day in the ophthalmology clinic. Other educational activities on the rotation included a skill session on the eye examination and a midclerkship feedback meeting with the clerkship director. After the mandate in March 2020 to withdraw medical students from all in-person clinical activities, all discussion sessions were hosted online via Zoom videoconferencing software (Zoom Video Communications, San Jose, $\mathrm{CA}$ ). There was no change to the content of the prerecorded lectures or the daily group discussion questions. Students were no longer permitted to participate in clinics, although the physical examination skills session and midclerkship meetings were still maintained in an adapted 
virtual format over Zoom. Students were asked to practice skills such as visual acuity testing, confrontation visual fields, pupil assessment, and drop instillation at home with a willing partner. These skills were then observed and discussed over Zoom with the clerkship director. The virtual physical examination skills session also incorporated video tutorials about direct ophthalmoscopy, followed by self-assessment questions and fundus image interpretation exercises. Additional virtual content, including interactive content and educational and surgical videos, were assigned in lieu of clinical experiences.

\section{Study Design}

All students who completed the ophthalmology clerkship were administered a 20-question survey, which is shown in -Table 1. This questionnaire was designed to assess the learner's perspective on the flipped classroom model, their interest in the field of ophthalmology, and their confidence in evaluating a variety of ophthalmic complaints in the primary care setting. A series of statements regarding these issues were presented to the student alongside a 6-point Likert scale. Students were asked to choose their level of agreement or disagreement, with 6 indicating the strongest level of agreement. Two yes or no questions were asked to assess whether the course enhanced the student's interest in ophthalmology, as well as the student's consideration of a career in ophthalmology. The survey was administered in a paper format on the last day of the in-person rotation and on the Qualtrics online survey site (Qualtrics, Provo, UT) in a time frame 1 week to 3 months after completing the online rotation. Both versions of the survey were identical in the questions that were asked.

This study was approved by the Institutional Review Board and deemed an exemption from consent. All student participation was voluntary, and students were informed that their answers would have no bearing on their grade or performance in the rotation. No identifying information was collected from the students and full anonymity was maintained.

\section{Statistical Analysis}

All available data were included in the statistical analysis, which was performed using IBM SPSS statistics software (Armonk, NY). Descriptive statistics were used to determine survey data means, and Student's two-tailed $t$-tests were used to compare survey responses between the in-person cohort and the online cohort. To further analyze the proportion of students' responses, Likert responses "strongly agree," "agree," and "somewhat agree" were grouped together into one cohort; likewise, Likert responses "strongly disagree," "disagree," and "somewhat disagree" were consolidated into another cohort. This created dichotomous categorical variables, which were compared using Fisher's exact test. Fisher's exact test was also used to compare survey responses between different grade levels. Statistical significance was set at $p<0.05$. As these data were a convenience-based sample, no calculations of power were performed.

\section{Results}

Between July 2019 and March 2020, 82 students completed the in-person ophthalmology rotation; of those, 68 students completed the survey, including 6 second-year students, 16 third-year students, and 44 fourth-year students. Between March 2020 and July 2020, 66 students completed the online ophthalmology rotation; of those, 44 students completed the survey, including 5 second-year students, 15 third-year students, and 24 fourth-year students. There was no statistical difference in the proportion of student grade levels among cohorts $(p=0.20)$, nor were there any significant differences in survey responses between junior (defined as second- and third-year students) and senior medical students.

Compared with students who participated in the inperson clerkship, students who participated in the online virtual clerkship were more likely to agree that the flipped classroom provides opportunities to think critically and is efficient ( - Table $\mathbf{1}$ ). These students were less likely to feel comfortable using the direct ophthalmoscope, and less likely to desire additional resident teaching sessions. However, students who completed the online clerkship were no less likely to feel comfortable with triaging eye complaints, evaluating acute vision loss, or evaluating eye pain (-Table 1). Compared with students in the in-person clerkship, a significantly higher proportion of students in the online clerkship reported that the course enhanced their interest in ophthalmology (-Table 1). However, a significantly lower proportion of students in the online cohort reported a career interest in the field of ophthalmology compared with students in the in-person cohort (-Table 1). Statistical analysis of the binned cohorts using Fisher's exact test yielded similar results and significances; as such, representative data are included in - Table 1.

Examination scores for both cohorts were also compared: for the in-person group, average examination score was $84.9 \%$; for the online group, the average examination score was $84.6 \%$. There was no statistically significant difference between the groups $(p=0.79)$.

\section{Discussion}

In this unprecedented global pandemic, medical educators must find novel ways to adapt to new social distancing guidelines without hampering the instruction of future physicians and other health care professionals. In situations where students are unable to meet face-to-face with peers, faculty, or patients to have a full in-person clinical experience, it is uncertain how to best maintain high-quality medical student education while protecting the safety of all involved. Moreover, with no clear end point to the pandemic, it is evident that we must act quickly to maximize learning and create sustainable solutions to the current challenges we face. Fortunately, the advancement of available technology paired with the creativity and innovation of instructors have allowed many to rise to the occasion, and the transition to remote learning has been widely embraced 
Table 1 Comparison of in-person and online classroom questionnaire responses after completing the ophthalmology flipped classroom clerkship

\begin{tabular}{|c|c|c|c|}
\hline Likert scales & $\begin{array}{l}\text { In-person } \\
\text { Mean (SD) } \\
n=68\end{array}$ & $\begin{array}{l}\text { Online } \\
\text { Mean (SD) } \\
n=44\end{array}$ & $p$-Value ${ }^{a}$ \\
\hline $\begin{array}{l}\text { I favor the use of a case-based flipped classroom over a traditional lecture- } \\
\text { based lecture format. }\end{array}$ & $4.18 \pm 1.22$ & $4.48 \pm 1.05$ & 0.181 \\
\hline Case-based flipped classrooms provide ample opportunity for me to learn. & $4.69 \pm 0.80$ & $5.02 \pm 0.70$ & 0.026 \\
\hline $\begin{array}{l}\text { Case-based flipped classrooms provide ample opportunity to think } \\
\text { critically } \\
\text { about clinical diagnoses and treatment. }\end{array}$ & $4.78 \pm 0.91$ & $4.95 \pm 0.71$ & 0.284 \\
\hline Case-based flipped classrooms are an efficient method of learning. & $4.46 \pm 1.24$ & $4.91 \pm 0.88$ & 0.038 \\
\hline Case-based flipped classrooms improve communication skills. & $4.49 \pm 1.10$ & $4.41 \pm 0.73$ & 0.658 \\
\hline There is more pressure to perform in case-based flipped classrooms. & $4.21 \pm 1.14$ & $4.11 \pm 1.20$ & 0.683 \\
\hline $\begin{array}{l}\text { Working in a case-based flipped classroom environment creates too much } \\
\text { burden. }\end{array}$ & $2.82 \pm 0.99$ & $2.52 \pm 1.11$ & 0.138 \\
\hline Flipped classrooms make me feel anxious. & $2.81 \pm 1.14$ & $2.95 \pm 1.26$ & 0.526 \\
\hline $\begin{array}{l}\text { Core medical topics should be taught using case-based flipped classroom } \\
\text { lectures. }\end{array}$ & $3.81 \pm 1.10$ & $4.14 \pm 1.13$ & 0.130 \\
\hline $\begin{array}{l}\text { Medical subspecialties should be taught using case-based flipped } \\
\text { classroom formats. }\end{array}$ & $4.13 \pm 1.04$ & $4.43 \pm 0.82$ & 0.113 \\
\hline I feel comfortable triaging a patient with an eye complaint. & $4.34 \pm 0.79$ & $4.52 \pm 0.59$ & 0.200 \\
\hline I feel comfortable using a direct ophthalmoscope. & $4.19 \pm 1.03$ & $2.70 \pm 1.15$ & $<0.001$ \\
\hline I am confident in evaluating a patient who presents with acute vision loss. & $4.33 \pm 0.79$ & $4.39 \pm 0.75$ & 0.700 \\
\hline I am confident in evaluating a patient who presents with eye pain. & $4.34 \pm 0.71$ & $4.39 \pm 0.69$ & 0.752 \\
\hline I am interested in the field of ophthalmology. & $3.31 \pm 1.25$ & $2.91 \pm 1.24$ & 0.096 \\
\hline $\begin{array}{l}\text { How often (what per cent of the time) would you like to see the flipped } \\
\text { classroom format used in medical education? }\end{array}$ & $60.2 \% \pm 16 \%$ & $65.0 \% \pm 16.8 \%$ & 0.128 \\
\hline I found the physical examination skill session useful. & $4.69 \pm 0.94$ & $4.32 \pm 1.18$ & 0.071 \\
\hline \multirow{2}{*}{$\begin{array}{l}\text { The physical examination skill session should include more than one } \\
\text { session. }\end{array}$} & $4.15 \pm 1.21$ & $3.30 \pm 1.17$ & $<0.001$ \\
\hline & $\begin{array}{l}\text { In-person } \\
\text { Answered "yes" }\end{array}$ & $\begin{array}{l}\text { Online } \\
\text { Answered "yes" }\end{array}$ & $p$-Value ${ }^{a}$ \\
\hline Did this course enhance interest in ophthalmology? & $79.1 \%(53)$ & $86.3 \%(38)$ & $<0.001$ \\
\hline Are you considering a career in ophthalmology? & $23.9 \%(16)$ & $4.5 \%(2)$ & $<0.001$ \\
\hline
\end{tabular}

Abbreviation: SD, standard deviation.

${ }^{a} p$-Value calculated via Student's two-tailed $t$-test.

by the medical education community. In fact, many recent studies are finding that online learning comes with a host of advantages over traditional learning models, including higher levels of student engagement. ${ }^{11}$

Our work suggests that the use of a flipped classroom model for an introductory ophthalmology clerkship may be successful even with the physical distancing constraints of the COVID era. As this model utilizes prerecorded lectures delivered electronically and synchronous discussions that can be shared over video conferencing, our flipped classroom model already had the infrastructure in place to function fluidly in an entirely online format. Additionally, the transition to digital learning was well supported by our institutional resources and allowed for comparison of student cohorts before and after this transition. These survey results demonstrate that, regardless of its mode of delivery, the case-based flipped classroom model appears to effectively impart the appropriate level of ophthalmic didactic knowledge and enhance student engagement with the field. This is further supported by the equivalence in examination grades between cohorts. Interestingly, after the curriculum was moved to the online format, a larger proportion of students reported that the course enhanced their interest in ophthalmology, while a smaller proportion of students declared that they were considering ophthalmology as a career. This may be a reflection of a difference in timing of the course throughout the academic year, as students tend to narrow their career interests over time, or it may reflect an 
imbalanced interest in ophthalmology across cohorts even prior to starting the rotation. As such, it is important to be aware of the potential bias in the results that may be present.

Our findings align well with work in other surgical subspecialties such as otolaryngology and urology that show online formats are effective in delivering didactic information to students. ${ }^{12,13}$ Within the field of ophthalmology, it has been evident that virtual models of learning implemented due to COVID-19 have been largely successful in execution, with high levels of student satisfaction and academic performance at multiple institutions. ${ }^{14,15}$ This study is unique among other reports of virtual curricula in that our institution had been analyzing the medical student experience of the flipped ophthalmology classroom prior to the COVID-19 pandemic. As such, this allowed a direct, quantifiable comparison to be made between students participating in the course before and after the shift to online learning.

The vast majority of students completed the rotation feeling confident about their ability to evaluate and triage patients presenting with eye complaints. However, unsurprisingly, students who participated in the online rotation reported significantly lower levels of confidence using the direct ophthalmoscope compared with students who spent time in the clinic. This highlights an inherent weakness of digital learning-the inability to replicate physical examination skills with specialized equipment. This is further supported by the finding that students in the online cohort were significantly less interested in participating in more than one physical examination skills session, which is perhaps an indication that the virtual format is less engaging or less effectual than a real-life experience. Alternately, the virtual physical examination skills session provided 1:1 feedback, compared with the small-group format of an in-person skills session, and students may have found this sufficient. Without the opportunity to rotate through ophthalmology clinics, it is entirely possible for the student to complete the rotation without ever having laid eyes or hands on a slit lamp. Remote learning may be an effective way to deliver didactic material and discussion sessions but will never be able to fully replace time in the clinic or operating room.

This study is not without limitations. As this study was only performed at a single department, there is inherent lack of curricular diversity, and it is difficult to know the generalizability of this model across different subspecialties, academic institutions, and levels of training. Additionally, the relatively small sample sizes surveyed in this study further limit the generalizability of our results. Another limitation lies in the fact that, as a survey-based study, our results reflect the subjective opinions and emotions of the students surveyed, with limited objective data outside of final examination scores. Moreover, the administration of the surveys themselves was inherently different between cohorts, both in questionnaire delivery (paper vs. online) and timing (immediately after the rotation vs. weeks or months later). This inconsistency was a variable that is uncontrolled for. Finally, as the transition to online learning was mandated in the face of a global pandemic, there are undoubtedly a host of confounding factors that may interfere with the comparabil- ity of the two cohorts, such as differences in students' mental well-being or their psychological response to remote learning.

Despite these limitations, this study supports the online delivery of the flipped classroom curriculum. Further studies should work to validate the efficacy of this model in a variety of settings and contexts. The implications of these results are significant as well. If the online flipped classroom continues to prove valuable, this may allow for the delivery of ophthalmology education to medical schools lacking an ophthalmology program, even long after the pandemic has quelled. This would allow all medical students to learn basic ophthalmological skills at a primary care level and potentially engage students lacking a home ophthalmology program. Future directions might focus on incorporating learners into telehealth encounters with ophthalmology patients or exploring video livestreams of surgical procedures to further expand the potential for remote learning-work that has been successfully piloted last year by Tsui et al. ${ }^{16}$

\section{Conclusion}

In the midst of vast uncertainty, medical students and educators have had to exercise resilience and adaptability during the COVID-19 pandemic to continue learning and teaching in a variety of novel formats. The flipped classroom model for the ophthalmology rotation appears readily translatable to an online modality, with the ability to adequately deliver a meaningful ophthalmology curriculum and foster engagement among its students. While it cannot fully replace certain aspects of in-person clinical learning, it may be a useful tool to either approximate or augment in-person rotations moving forward.

\section{Disclosure}

No benefits in any form have been or will be received from any commercial party related directly or indirectly to the subject of this manuscript.

\section{Conflict of Interest}

None declared.

\section{References}

1 Whelan A, Young G, Catanese VM. Guidance on Medical Students' Clinical Participation: Effective Immediately. Association of American Medical Colleges; 2020

2 Chen CH, Mullen AJ. COVID-19 can catalyze the modernization of medical education. JMIR Med Educ 2020;6(01):e19725

3 Chen KS, Monrouxe L, Lu YH, et al. Academic outcomes of flipped classroom learning: a meta-analysis. Med Educ 2018;52(09): 910-924

4 Hew KF, Lo CK. Flipped classroom improves student learning in health professions education: a meta-analysis. BMC Med Educ 2018;18(01):38

5 Tu DC, Park D-W, Krisciunas T, Hwang TS. Teaching ophthalmology residents clinical optics via a flipped classroom curriculum. J Acad Ophthalmol 2019;11(01):e16-e21

6 Alabiad CR, Moore KJ, Green DP, Kofoed M, Mechaber AJ, Karp CL. The flipped classroom: an innovative approach to medical 
education in ophthalmology. J Acad Ophthalmol 2020;12(02): e96-e103

7 Chick RC, Clifton GT, Peace KM, et al. Using technology to maintain the education of residents during the COVID-19 pandemic. J Surg Educ 2020;77(04):729-732

8 Ding C, Li S, Chen B. Effectiveness of flipped classroom combined with team-, case-, lecture- and evidence-based learning on ophthalmology teaching for eight-year program students. BMC Med Educ 2019;19(01):419

9 Diel RJ, Avdic A, Kemp PS. Flipped ophthalmology classroom: a better way to teach medical students. J Acad Ophthalmol 2020;12 (02):e104-e109

10 Allen RC, Harper RAAmerican Academy of Ophthalmology. Basic Ophthalmology: Essentials for Medical Students. 10th ed. San Francisco, CA: American Academy of Ophthalmology; 2016

11 Kay D, Pasarica M. Using technology to increase student (and faculty satisfaction with) engagement in medical education. Adv Physiol Educ 2019;43(03):408-413
12 Steehler AJ, Pettitt-Schieber B, Studer MB, Mahendran G, Pettitt BJ, Henriquez OA. Implementation and evaluation of a virtual elective in otolaryngology in the time of COVID-19. Otolaryngol Head Neck Surg 2020:194599820951150

13 Williams C, Familusi OO, Ziemba J, et al. Adapting to the educational challenges of a pandemic: development of a novel virtual urology sub-internship during the time of COVID-19. Urology 2021;148:70-76

14 DeVaro SN, Uner OE, Khalifa YM, Graubart EB. Ophthalmology education in COVID-19: a remote elective for medical students. J Acad Ophthalmol 2020;12(02):e165-e170

15 Scanzera AC, Cole E, Valikodath N, et al. Implementation of COVID19 protocols and tele-triage in an academic ophthalmology department. J Acad Ophthalmol 2020;12(02):e151-e158

16 Tsui E, Wells MB, Mohamed M, Felix CM, Giaconi JA. Virtual ophthalmology rotations-a real possibility during the COVID19 pandemic and beyond. J Acad Ophthalmol 2020;12(02): e195-e199 